Sharif University of Technology
Scientia Iranica
Transactions E: Industrial Engineering
http://scientiairanica.sharif.edu

\title{
Robust aggregation operators for multi-criteria decision-making with intuitionistic fuzzy soft set environment
}

\author{
R. Arora and H. Garg* \\ School of Mathematics, Thapar Institute of Engineering \& Technology (Deemed University), Patiala 147004, Punjab, India.
}

Received 6 September 2016; received in revised form 5 October 2016; accepted 28 January 2017

\section{KEYWORDS}

Fuzzy soft sets;

Intuitionistic fuzzy

soft sets;

Decision-making;

Aggregation

operators.

\begin{abstract}
Soft set theory acts as a fundamental tool for handling the uncertainty in the data by adding a parameterized factor during the process unlike fuzzy as well as intuitionistic fuzzy set theory. In this manuscript, an attempt has been made to compare two Intuitionistic Fuzzy Soft Numbers (IFSNs) and then weighted averaging and geometric aggregation operators for aggregating the different input arguments have been presented. Further, their various properties have been established. The effectiveness of these operators has been demonstrated through a case study.
\end{abstract}

(C) 2018 Sharif University of Technology. All rights reserved.

\section{Introduction}

Molodtsov [1] initially presented the concept of Soft Set (SS) theory as a fundamental tool for modeling the uncertainty and vague data. Various traditional tools, including, but not limited to, Fuzzy Set (FS) theory [2], Intuitionistic Fuzzy Set (IFS) theory [3] etc., have been widely used by the researchers [415] to handle the uncertainties during the analysis. However, all these techniques lack parameterizations tool and hence they cannot be effectively applied in real life problems. To handle these issues, SS theory pays a great attention to and successfully deals with these types of conditions. Maji et al. [16] and Maji et al. [17] combined the theory of SS with the fuzzy and intuitionistic fuzzy set theories and called them Fuzzy Soft Set (FSS) and Intuitionistic Fuzzy Soft Set (IFSS), respectively. Meanwhile, the study of hybrid models by combining SS with the others has

\footnotetext{
*. Corresponding author.

E-mail address: harishg58iitr@gmail.com (H. Garg)
}

been developed such as on fuzzy soft set $[18,19]$, intuitionistic fuzzy soft sets $[16,20]$, generalized fuzzy soft set $[21,22]$, generalized intuitionistic fuzzy soft set [2326], fuzzy number intuitionistic fuzzy soft sets [27], etc. [28-33]. Furthermore, various researchers [34-37] have proposed several types of distance and similarity measures under FSS and/or IFSS environments. Roy and Maji [38] presented an approach to decision making problems under FSS environment. Yang et al. [33] analyzed the problem of decision making by introducing the concept of interval-valued soft sets.

Thus, by considering the fact that the IFSS is a powerful tool to deal with the ambiguity and vagueness of the data, the present paper will introduce new averaging/geometric intuitionistic fuzzy soft aggregation operators based on intuitionistic fuzzy soft numbers (IFSNs). Their advantage is being capable to facilitate the descriptions of the real-world situation with the help of their parameterizations property. In order to rank the different alternatives, having an aggregation operator is necessary to aggregate all the preferences of the decision maker into a collective value and hence to find a desirable alternative(s) according 
to the score values. However, to the best of our knowledge, the research on FSS and IFSS is limited to their basic theories and applications and there is no research on their aggregation information. Therefore, it is necessary to investigate this issue; we will propose some suitable operators for aggregating the preferences of the decision makers. Various desirable properties of the proposed operators have also been investigated in details. Furthermore, an approach for Multi-Criteria Decision Making (MCDM) method has been discussed based on these operators and their effectiveness has been explained through a case study of medical diagnosis, where the most desirable alternative has been identified under the set of different criteria. Finally, the computed results are compared with the results of the existing operators to show their effectiveness.

The rest of the manuscript is organized as follows. Section 2 presents some basic concepts on FSS and IFSSs. Some averaging/geometric aggregation operators are presented in Section 3. Section 4 discusses the MCDM approach based on the proposed operators for IFSNs, followed by an illustrative example. Finally, a conclusion has been drawn in Section 5 .

\section{Basic concepts on FSS and IFSS}

Let $U$ be the universal set and $E$ be a set of parameters then the soft set over $U[1]$ is defined as the pair $(F, E)$ where $F: E \rightarrow K^{U}, K^{U}$ is the set of all subsets of $U$. Further, this pair is called FSS [16] over $U$ iff $F$ : $E \rightarrow I^{U}, I^{U}$ is the set of all fuzzy subsets of $U$, which is defined as $F_{e_{j}}=\left\{\left\langle x, \mu_{j}(x)\right\rangle \mid x \in U\right\}$. For any parameter $e_{j}$, FSS reduces to SS if $F_{e_{j}}$ is a crisp subset of $U$.

\section{Example 2.1.}

Let $U=\left\{h_{1}, h_{2}, h_{3}, h_{4}\right\}$ be a set of four houses and $E=\left\{\right.$ "expensive $\left(e_{1}\right)$ ", "wooden $\left(e_{2}\right)$ ", "cheap $\left(e_{3}\right)$ ", "beautiful $\left(e_{4}\right)$ ", "in good location $\left(e_{5}\right)$ " $\}$ be a set of parameters; then, the FSS for describing the "attractiveness of the houses" is $(F, E)=\left\{F_{e_{1}}, F_{e_{2}}, F_{e_{3}}\right.$, $\left.F_{e_{4}}, F_{e_{5}}\right\}$, where $F_{e_{1}}=\left\{\left\langle\left(h_{2}, 0.2\right),\left(h_{3}, 0.7\right)\right\rangle\right\}, F_{e_{2}}=$ $\left\{\left\langle\left(h_{1}, 0.6\right),\left(h_{3}, 0.7\right),\left(h_{4}, 0.9\right)\right\rangle\right\}, F_{e_{3}}=\left\{\left\langle\left(h_{1}, 0.3\right),\left(h_{4}\right.\right.\right.$, $0.5)\rangle\}, F_{e_{4}}=\left\{\left\langle\left(h_{1}, 0.6\right),\left(h_{2}, 0.9\right),\left(h_{3}, 0.7\right)\right\rangle\right\}$ and $F_{e_{5}}$ $=\left\{\left\langle\left(h_{2}, 0.7\right),\left(h_{4}, 0.6\right)\right\}\right.$.

On the other hand, a pair $(F, E)$ is called an IFSS [16] over $U$ iff $F: E \rightarrow I F S(U)$, where $I F S(U)$ denotes the set of all intuitionistic fuzzy subsets of $U$ and is defined as $F_{e_{j}}\left(x_{i}\right)=\left\{\left\langle x_{i}, \mu_{j}\left(x_{i}\right), \nu_{j}\left(x_{i}\right)\right\rangle \mid\right.$ $\left.x_{i} \in U\right\}$ for any parameter $e_{j} \in E$, where $\mu_{j}\left(x_{i}\right)$ and $\nu_{j}\left(x_{i}\right)$ are degrees of acceptance and rejection, respectively, with the conditions $0 \leq \mu_{j}\left(x_{i}\right), \nu_{j}\left(x_{i}\right) \leq 1$ and $\mu_{j}\left(x_{i}\right)+\nu_{j}\left(x_{i}\right) \leq 1$.

\section{Example 2.2.}

Consider the above example of "attractiveness of the houses" under the same parameter; IFSS can be written as:

$$
(F, E)=\left\{F_{e_{1}}, F_{e_{2}}, F_{e_{3}}, F_{e_{4}}, F_{e_{5}}\right\},
$$

where:

$$
\begin{aligned}
& F_{e_{1}}=\left\{\left\langle\left(h_{2}, 0.2,0.4\right),\left(h_{3}, 0.7,0.1\right)\right\rangle\right\}, \\
& F_{e_{2}}=\left\{\left\langle\left(h_{1}, 0.6,0.3\right),\left(h_{3}, 0.7,0.2\right),\left(h_{4}, 0.9,0.1\right)\right\rangle\right\}, \\
& F_{e_{3}}=\left\{\left\langle\left(h_{1}, 0.3,0.5\right),\left(h_{4}, 0.5,0.3\right)\right\rangle\right\}, \\
& F_{e_{4}}=\left\{\left\langle\left(h_{1}, 0.6,0.3\right),\left(h_{2}, 0.9,0.0\right),\left(h_{3}, 0.7,0.2\right)\right\rangle\right\}, \\
& F_{e_{5}}=\left\{\left\langle\left(h_{2}, 0.7,0.2\right),\left(h_{4}, 0.6,0.2\right)\right\rangle\right\} .
\end{aligned}
$$

For the sake of simplicity, we denote the pair of $F_{e_{j}}\left(x_{i}\right)=\left\{\left\langle x_{i}, \mu_{j}\left(x_{i}\right), \nu_{j}\left(x_{i}\right)\right\rangle \mid x_{i} \in U\right\}$ to be $F_{e_{i j}}=\left\langle\mu_{i j}, \nu_{i j}\right\rangle$ and call it intuitionistic fuzzy soft number (IFSN). In the process of applying IFSNs to practical problems, it is necessary to rank them. For this, a score function of $F_{e_{i j}}$ is defined as [39]:

$$
S\left(F_{e_{i j}}\right)=\mu_{i j}-\nu_{i j}
$$

where $S\left(F_{e_{i j}}\right) \in[-1,1]$. In this definition, it can be seen that the larger $S\left(F_{e_{i j}}\right)$, the larger is IFSN $F_{e_{i j}}$.

\section{Example 2.3.}

Let $F_{e_{11}}=\langle 0.4,0.2\rangle$ and $F_{e_{12}}=\langle 0.3,0.5\rangle$ be two IFSNs; then, by using Eq. (1), we get $S\left(F_{e_{11}}\right)=0.2$ and $S\left(F_{e_{12}}\right)=-0.2$. Since $S\left(F_{e_{11}}\right)>S\left(F_{e_{12}}\right)$, we have $F 6_{e_{11}} \succ F_{e_{12}}$, where " $\succ$ " refers to "preferred to".

However, in some situations, the above function cannot be used to compare IFSNs. For example, let $F_{e_{11}}=\langle 0.2,0.4\rangle$ and $F_{e_{12}}=\langle 0.3,0.5\rangle$; then, it is impossible to know which one is bigger because $S\left(F_{e_{11}}\right)=S\left(F_{e_{12}}\right)$. For this, an accuracy function $H$ of $F_{e_{i j}}$ is defined as [39]:

$$
H\left(F_{e_{i j}}\right)=\mu_{i j}+\nu_{i j}
$$

where $H\left(F_{e_{i j}}\right) \in[0,1]$. Thus, in order to compare two IFSNs $F_{e_{i j}}$ and $G_{e_{i j}}$, the following ranking and comparison laws for two IFSNs are defined:

(i) If $S\left(F_{e_{i j}}\right)>S\left(G_{e_{i j}}\right)$, then $F_{e_{i j}} \succ G_{e_{i j}}$;

(ii) If $S\left(F_{e_{i j}}\right)=S\left(G_{e_{i j}}\right)$, then:

- If $H\left(F_{e_{i j}}\right)>H\left(G_{e_{i j}}\right)$ then $F_{e_{i j}} \succ G_{e_{i j}}$;

- If $H\left(F_{e_{i j}}\right)=H\left(G_{e_{i j}}\right)$ then $F_{e_{i j}}=G_{e_{i j}}$. 


\section{Aggregation operators for intuitionistic fuzzy soft numbers}

In this section, an aggregation operators, namely, intuitionistic fuzzy soft weighted average and intuitionistic fuzzy soft weighted geometric, for intuitionistic fuzzy soft numbers have been presented.

\subsection{Operational laws for IFSNs}

Definition 3.1. Let $F_{e}=\langle\mu, \nu\rangle, F_{e_{11}}=\left\langle\mu_{11}, \nu_{11}\right\rangle$; $F_{e_{12}}=\left\langle\mu_{12}, \nu_{12}\right\rangle$ be three IFSNs; for any real number $\lambda>0$, by algebraic norms, we have:

(i) $F_{e_{11}} \oplus F_{e_{12}}=\left\langle\mu_{11}+\mu_{12}-\mu_{11} \mu_{12}, \nu_{11} \nu_{12}\right\rangle$;

(ii) $F_{e_{11}} \otimes F_{e_{12}}=\left\langle\mu_{11} \mu_{12}, \nu_{11}+\nu_{12}-\nu_{11} \nu_{12}\right\rangle$;

(iii) $\lambda F_{e}=\left\langle 1-(1-\mu)^{\lambda}, \nu^{\lambda}\right\rangle$;

(iv) $F_{e}^{\lambda}=\left\langle\mu^{\lambda}, 1-(1-\nu)^{\lambda}\right\rangle$.

Based on these laws, some averaging and geometric aggregation operators, for a collections of IFSNs denoted by $\Omega$, have been defined here.

\subsection{Intuitionistic Fuzzy Soft Weighted Average (IFSWA) operator}

Definition 3.2. Let $F_{e_{i j}}=\left\langle\mu_{i j}, \nu_{i j}\right\rangle,(i=1,2, \ldots$, $n ; j=1,2, \ldots, m)$ be an IFSNs and $\eta_{j}, \xi_{i}$ be the weight vectors for the parameters $e_{j}$ 's and experts $x_{i}$, respectively; satisfying $\eta_{j}>0, \xi_{i}>0$ and $\sum_{j=1}^{m} \eta_{j}=1$, $\sum_{i=1}^{n} \xi_{i}=1, \operatorname{IFSWA}: \Omega^{n} \longrightarrow \Omega$ operator is defined as:

$$
\operatorname{IFSWA}\left(F_{e_{11}}, F_{e_{12}}, \ldots, F_{e_{n m}}\right)=\bigoplus_{j=1}^{m} \eta_{j}\left(\bigoplus_{i=1}^{n} \xi_{i} F_{e_{i j}}\right)
$$

Theorem 3.1. Let $F_{e_{i j}}=\left\langle\mu_{i j}, \nu_{i j}\right\rangle, i=1,2, \ldots$, $n ; j=1,2, \ldots, m$, be an IFSNs; the aggregated value by IFSWA operator is also an IFSN, and given by:

$$
\begin{aligned}
& I F S W A\left(F_{e_{11}}, F_{e_{12}}, \ldots, F_{e_{n m}}\right) \\
& =\left\langle 1-\prod_{j=1}^{m}\left(\prod_{i=1}^{n}\left(1-\mu_{i j}\right)^{\xi_{i}}\right)^{\eta_{j}}, \prod_{j=1}^{m}\left(\prod_{i=1}^{n} \nu_{i j}^{\xi_{i}}\right)^{\eta_{j}}\right\rangle .
\end{aligned}
$$

Proof. For $n=1$, we get $\xi_{1}=1$. Thus, by using an operations law defined in Definition 3.1, we have:

$$
\begin{array}{rl}
I F & S W A\left(F_{e_{11}}, F_{e_{12}}, \ldots, F_{e_{1 m}}\right)=\bigoplus_{j=1}^{m} \eta_{j} F_{e_{1 j}} \\
& =\left\langle 1-\prod_{j=1}^{m}\left(1-\mu_{1 j}\right)^{\eta_{j}}, \prod_{j=1}^{m} \nu_{1 j}^{\eta_{j}}\right\rangle \\
& =\left\langle 1-\prod_{j=1}^{m}\left(\prod_{i=1}^{1}\left(1-\mu_{i j}\right)^{\xi_{i}}\right)^{\eta_{j}}, \prod_{j=1}^{m}\left(\prod_{i=1}^{1} \nu_{i j}^{\xi_{i}}\right)^{\eta_{j}}\right\rangle .
\end{array}
$$

For $m=1$, we get $\eta_{1}=1$; thus, we have:

$$
\begin{aligned}
I F S W A\left(F_{e_{11}}, F_{e_{21}}, \ldots, F_{e_{n 1}}\right)=\bigoplus_{i=1}^{n} \xi_{i} F_{e_{i 1}} \\
=\left\langle 1-\prod_{i=1}^{n}\left(1-\mu_{i 1}\right)^{\xi_{i}}, \prod_{i=1}^{n} \nu_{i 1}^{\xi_{i}}\right\rangle \\
=\left\langle 1-\prod_{j=1}^{1}\left(\prod_{i=1}^{n}\left(1-\mu_{i j}\right)^{\xi_{i}}\right)^{\eta_{j}},\right. \\
\left.\prod_{j=1}^{1}\left(\prod_{i=1}^{n} \nu_{i j}^{\xi_{i}}\right)^{\eta_{j}}\right\rangle .
\end{aligned}
$$

Thus, the results are true for $n=1$ and $m=1$. Assume the results hold for $m=k_{1}+1, n=k_{2}$ and $m=k_{1}, n=$ $k_{2}+1$, i.e.:

$$
\begin{aligned}
\bigoplus_{j=1}^{k_{1}+1} \eta_{j}\left(\bigoplus_{i=1}^{k_{2}} \xi_{i} F_{e_{i j}}\right)= & \left\langle 1-\prod_{j=1}^{k_{1}+1}\left(\prod_{i=1}^{k_{2}}\left(1-\mu_{i j}\right)^{\xi_{i}}\right)^{\eta_{j}}\right. \\
& \left.\prod_{j=1}^{k_{1}+1}\left(\prod_{i=1}^{k_{2}} \nu_{i j}^{\xi_{i}}\right)^{\eta_{j}}\right\rangle
\end{aligned}
$$

and:

$$
\begin{aligned}
\bigoplus_{j=1}^{k_{1}} \eta_{j}\left(\bigoplus_{i=1}^{k_{2}+1} \xi_{i} F_{e_{i j}}\right)= & \left\langle 1-\prod_{j=1}^{k_{1}}\left(\prod_{i=1}^{k_{2}+1}\left(1-\mu_{i j}\right)^{\xi_{i}}\right)^{\eta_{j}}\right. \\
& \left.\prod_{j=1}^{k_{1}}\left(\prod_{i=1}^{k_{2}+1} \nu_{i j}^{\xi_{i}}\right)^{\eta_{j}}\right\rangle
\end{aligned}
$$

Now, for $m=k_{1}+1, n=k_{2}+1$, we get:

$$
\bigoplus_{j=1}^{k_{1}+1} \eta_{j}\left(\bigoplus_{i=1}^{k_{2}+1} \xi_{i} F_{e_{i j}}\right)=\bigoplus_{j=1}^{k_{1}+1} \eta_{j}
$$$$
\left(\bigoplus_{i=1}^{k_{2}} \xi_{i} F_{e_{i j}} \oplus \xi_{k_{2}+1} F_{e_{\left(k_{2}+1\right) j}}\right)
$$$$
=\bigoplus_{j=1}^{k_{1}+1} \bigoplus_{i=1}^{k_{2}} \eta_{j} \xi_{i} F_{e_{i j}} \bigoplus_{j=1}^{k_{1}+1} \eta_{j} \xi_{k_{2}+1} F_{e_{\left(k_{2}+1\right) j}}
$$$$
=\left\langle 1-\prod_{j=1}^{k_{1}+1}\left(\prod_{i=1}^{k_{2}}\left(1-\mu_{i j}\right)^{\xi_{i}}\right)^{\eta_{j}} \oplus 1\right.
$$$$
-\prod_{j=1}^{k_{1}+1}\left(\left(1-\mu_{\left(k_{2}+1\right) j}\right)^{\xi_{k_{2}+1}}\right)^{\eta_{j}}
$$$$
\left.\prod_{j=1}^{k_{1}+1}\left(\prod_{i=1}^{k_{2}} \nu_{i j}^{\xi_{i}}\right)^{\eta_{j}} \oplus \prod_{j=1}^{k_{1}+1}\left(\nu_{\left(k_{2}+1\right) j}^{\xi_{k_{2}+1}}\right)^{\eta_{j}}\right\rangle
$$ 


$$
\begin{aligned}
= & \left\langle 1-\prod_{j=1}^{k_{1}+1}\left(\prod_{i=1}^{k_{2}+1}\left(1-\mu_{i j}\right)^{\xi_{i}}\right)^{\eta_{j}},\right. \\
& \left.\prod_{j=1}^{k_{1}+1}\left(\prod_{i=1}^{k_{2}+1} \nu_{i j}^{\xi_{i}}\right)^{\eta_{j}}\right\rangle .
\end{aligned}
$$

Thus, it is true for $m=k_{1}+1$ and $n=k_{2}+1$, and hence, by induction, the results holds for all $m, n \geq 1$.

Since $0 \leq \mu_{i j} \leq 1 \Leftrightarrow 0 \leq \prod_{i=1}^{n}\left(1-\mu_{i j}\right)^{\xi_{i}} \leq 1$ $\Leftrightarrow 0 \leq \prod_{j=1}^{m}\left(\prod_{i=1}^{n}\left(1-\mu_{i j}\right)^{\xi_{i}}\right)^{\eta_{j}} \leq 1$ and thus, $0 \leq$ $1-\prod_{j=1}^{m}\left(\prod_{i=1}^{n}\left(1-\mu_{i j}\right)^{\xi_{i}}\right)^{\eta_{j}} \leq 1$. On the other hand, $0 \leq$ $\nu_{i j} \leq 1 \Leftrightarrow 0 \leq \prod_{i=1}^{n} \nu_{i j}^{\xi_{i}} \leq 1 \Leftrightarrow 0 \leq \prod_{j=1}^{m}\left(\prod_{i=1}^{n} \nu_{i j}^{\xi_{i}}\right)^{\eta_{j}} \leq 1$. Finally, $1-\prod_{j=1}^{m}\left(\prod_{i=1}^{n}\left(1-\mu_{i j}\right)^{\xi_{i}}\right)^{\eta_{j}}+\prod_{j=1}^{m}\left(\prod_{i=1}^{n} \nu_{i j}^{\xi_{i}}\right)^{\eta_{j}} \leq$ $1-\prod_{j=1}^{m}\left(\prod_{i=1}^{n}\left(1-\mu_{i j}\right)^{\xi_{i}}\right)^{\eta_{j}}+\prod_{j=1}^{m}\left(\prod_{i=1}^{n}\left(1-\mu_{i j}\right)^{\xi_{i}}\right)^{\eta_{j}} \leq$ 1. Thus, the aggregated value obtained by IFSWA operator is again an IFSN.

Remark 3.1. If the only parameter is $e_{1}$, i.e., $m=1$, then IFSWA operator reduces to IFWA operator [39]:

$$
\begin{aligned}
I F W A & \left(F_{e_{11}}, F_{e_{21}}, \ldots, F_{e_{n 1}}\right) \\
& =\left\langle 1-\prod_{i=1}^{n}\left(1-\mu_{i 1}\right)^{\xi_{i}}, \prod_{i=1}^{n} \nu_{i 1}^{\xi_{i}}\right\rangle .
\end{aligned}
$$

Thus, it is concluded that the aggregation operator defined under IFS environment is taken as a special case of the proposed operator.

\section{Example 3.1.}

Let $X=\left\{x_{1}, x_{2}, x_{3}, x_{4}\right\}$ be the set of experts, who are going to describe the "attractiveness of a house" under the set of parameters $E=\left\{e_{1}, e_{2}, e_{3}\right\}$. The rating values of the experts are assumed to be given in the form of $\operatorname{IFSNs}(F, E)=\left\langle\mu_{i j}, \nu_{i j}\right\rangle_{4 \times 3}$ for each parameters, which are summarized as follows:

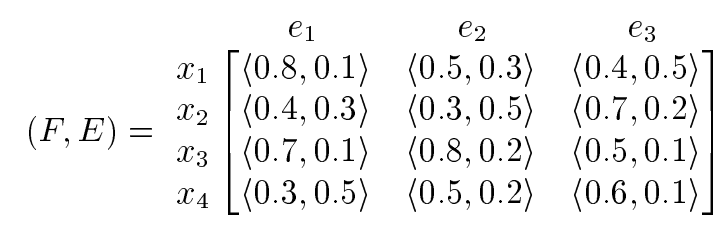

Let $\eta=(0.3,0.5,0.2)^{T}$ and $\xi=(0.1,0.2,0.4,0.3)^{T}$ be the weight vectors of the parameters and experts, respectively. By using Eq. (3), we get:

$$
\begin{aligned}
& I F S W A\left(F_{e_{11}}, F_{e_{12}}, \ldots, F_{e_{43}}\right) \\
& =\left\langle 1-\prod_{j=1}^{3}\left(\prod_{i=1}^{4}\left(1-\mu_{i j}\right)^{\xi_{i}}\right)^{\eta_{j}}\right. \\
& \left.\prod_{j=1}^{3}\left(\prod_{i=1}^{4} \nu_{i j}^{\xi_{i}}\right)^{\eta_{j}}\right\rangle=\left\langle 1-\left\langle\left\{(1-0.8)^{0.1}(1-0.4)^{0.2}\right.\right.\right. \\
& \left.(1-0.7)^{0.4}(1-0.3)^{0.3}\right\}^{0.3}\left\{(1-0.5)^{0.1}\right. \\
& \left.(1-0.3)^{0.2}(1-0.8)^{0.4}(1-0.5)^{0.3}\right\}^{0.5} \\
& \left\{(1-0.4)^{0.1}(1-0.7)^{0.2}(1-0.5)^{0.4}\right. \\
& \left.\left.(1-0.6)^{0.3}\right\}^{0.2}\right),\left(0.1^{0.1} 0.3^{0.2} 0.1^{0.4} 0.5^{0.3}\right)^{0.3} \\
& \left(0.3^{0.1} 0.5^{0.2} 0.2^{0.4} 0.2^{0.3}\right)^{0.5} \\
& \left.\left(0.5^{0.1} 0.2^{0.2} 0.1^{0.4} 0.1^{0.3}\right)^{0.2}\right\rangle \\
& =\langle 0.6017,0.2073\rangle \text {. }
\end{aligned}
$$

Based on Theorem 3.1, we have some properties of the proposed IFSWA operator for a collections of an IFSNs $F_{e_{i j}}=\left\langle\mu_{i j}, \nu_{i j}\right\rangle(i=1,2, \ldots, n ; j=1,2, \ldots, m)$.

Property 3.1 (Idempotency). If $F_{e_{i j}}=F_{e}=\langle\mu, \nu$ \rangle (say), for all $i, j$, then:

$$
I F S W A\left(F_{e_{11}}, F_{e_{12}}, \ldots, F_{e_{n m}}\right)=F_{e} .
$$

Proof. Since all $F_{e_{i j}}=F_{e}=\langle\mu, \nu\rangle$, then we have:

$$
\begin{aligned}
\operatorname{IFSWA}\left(F_{e_{11}}, F_{e_{12}}, \ldots, F_{e_{n m}}\right) & \left\langle 1-\prod_{j=1}^{m}\left(\prod_{i=1}^{n}(1-\mu)^{\xi_{i}}\right)^{\eta_{j}},\right. \\
& \left.\prod_{j=1}^{m}\left(\prod_{i=1}^{n} \nu^{\xi_{i}}\right)^{\eta_{j}}\right\rangle=\langle 1 \\
& \left.-\left((1-\mu)^{\sum_{i=1}^{n} \xi_{i}}\right)^{\sum_{j=1}^{m} \eta_{j}},\left(\nu^{\sum_{i=1}^{n} \xi_{i}}\right)^{\sum_{j=1}^{m} \eta_{j}}\right\rangle \\
= & \langle 1-(1-\mu), \nu\rangle=\langle\mu, \nu\rangle=F_{e} .
\end{aligned}
$$

Hence, proof is complete. 
Property 3.2 (Boundedness). Let $F_{e_{i j}}^{-}=\left\langle\min _{j}\right.$ $\left.\min _{i}\left\{\mu_{i j}\right\}, \max _{j} \max _{i}\left\{\nu_{i j}\right\}\right\rangle$ and $F_{e_{i j}}^{+}=\left\langle\max _{j} \max _{i}\left\{\mu_{i j}\right\}\right.$, $\left.\min _{j} \min _{i}\left\{\nu_{i j}\right\}\right\rangle ;$ then:

$$
F_{e_{i j}}^{-} \leq \operatorname{IFSWA}\left(F_{e_{11}}, F_{e_{12}}, \ldots, F_{e_{n m}}\right) \leq F_{e_{i j}}^{+} \text {. }
$$

Proof. As $F_{e_{i j}}=\left\langle\mu_{i j}, \nu_{i j}\right\rangle$ is an IFSN, then $\min _{j} \min _{i}$ $\left\{\mu_{i j}\right\} \leq \mu_{i j} \leq \max _{j} \max _{i}\left\{\mu_{i j}\right\}$; this implies that:

$$
\begin{aligned}
1 & -\max _{j} \max _{i}\left\{\mu_{i j}\right\} \leq 1-\mu_{i j} \leq 1-\min _{j} \min _{i}\left\{\mu_{i j}\right\} \\
\Leftrightarrow & \left(1-\max _{j} \max _{i}\left\{\mu_{i j}\right\}\right)^{\xi_{i}} \leq\left(1-\mu_{i j}\right)^{\xi_{i}} \\
\leq & \left(1-\min _{j} \min _{i}\left\{\mu_{i j}\right\}\right)^{\xi_{i}} \Leftrightarrow 1-\max _{j} \max _{i}\left\{\mu_{i j}\right\} \\
& \leq \prod_{i=1}^{n}\left(1-\mu_{i j}\right)^{\xi_{i}} \leq 1-\min _{j} \min _{i}\left\{\mu_{i j}\right\} \\
\Leftrightarrow & \left(1-\max _{j} \max _{i}\left\{\mu_{i j}\right\}\right)^{\sum_{j=1}^{m} \eta_{j}} \leq \prod_{j=1}^{m}\left(\prod_{i=1}^{n}\left(1-\mu_{i j}\right)^{\xi_{i}}\right)^{\eta_{j}} \\
& \leq\left(1-\min _{j} \min _{i}\left\{\mu_{i j}\right\}\right)^{\sum_{j=1}^{m} \eta_{j}},
\end{aligned}
$$

i.e.:

$$
\begin{aligned}
& \Leftrightarrow 1-\max _{j} \max _{i}\left\{\mu_{i j}\right\} \leq \prod_{j=1}^{m}\left(\prod_{i=1}^{n}\left(1-\mu_{i j}\right)^{\xi_{i}}\right)^{\eta_{j}} \\
& \leq 1-\min _{j} \min _{i}\left\{\mu_{i j}\right\} .
\end{aligned}
$$

Hence:

$$
\begin{aligned}
\min _{j} \min _{i}\left\{\mu_{i j}\right\} & \leq 1-\prod_{j=1}^{m}\left(\prod_{i=1}^{n}\left(1-\mu_{i j}\right)^{\xi_{i}}\right)^{\eta_{j}} \\
& \leq \max _{j} \max _{i}\left\{\mu_{i j}\right\} .
\end{aligned}
$$

On the other hand, $\min _{j} \min _{i}\left\{\nu_{i j}\right\} \leq \nu_{i j} \leq \max _{j} \max _{i}$ $\left\{\nu_{i j}\right\}$; this implies:

$$
\begin{aligned}
& \left(\min _{j} \min _{i}\left\{\nu_{i j}\right\}\right)^{\sum_{i=1}^{n}} \xi_{i} \leq \prod_{i=1}^{n} \nu_{i j}^{\xi_{i}} \leq\left(\max _{j} \max _{i}\left\{\nu_{i j}\right\}\right)^{\sum_{i=1}^{n} \xi_{i}} \\
& \Leftrightarrow \min _{j} \min _{i}\left\{\nu_{i j}\right\} \leq \prod_{i=1}^{n} \nu_{i j}^{\xi_{i}} \leq \max _{j} \max _{i}\left\{\nu_{i j}\right\} \\
& \Leftrightarrow\left(\min _{j} \min _{i}\left\{\nu_{i j}\right\}\right)^{\eta_{j}} \leq\left(\prod_{i=1}^{n} \nu_{i j}^{\xi_{i}}\right)^{\eta_{j}} \leq\left(\max _{j} \max _{i}\left\{\nu_{i j}\right\}\right)^{\eta_{j}} \\
& \Leftrightarrow\left(\min _{j} \min _{i}\left\{\nu_{i j}\right\}\right)^{\sum_{j=1}^{m}} \eta_{j} \leq \prod_{j=1}^{m}\left(\prod_{i=1}^{n} \nu_{i j}^{\xi_{i}}\right)^{\eta_{j}}
\end{aligned}
$$

$$
\leq\left(\max _{j} \max _{i}\left\{\nu_{i j}\right\}\right)^{\sum_{j=1}^{m} \eta_{j}},
$$

and hence, we have:

$$
\min _{j} \min _{i}\left\{\nu_{i j}\right\} \leq \prod_{j=1}^{m}\left(\prod_{i=1}^{n} \nu_{i j}^{\xi_{i}}\right)^{\eta_{j}} \leq \max _{j} \max _{i}\left\{\nu_{i j}\right\} .
$$

Let $\alpha \equiv I F S W A\left(F_{e_{11}}, F_{e_{12}}, \ldots, F_{e_{n m}}\right)=\left\langle\mu_{\alpha}, \nu_{\alpha}\right\rangle$; then, we have from Eqs. (4) and (5), $\min _{j} \min _{i}\left\{\mu_{i j}\right\} \leq$ $\mu_{\alpha} \leq \max _{j} \max _{i}\left\{\mu_{i j}\right\}$ and $\min _{j} \min _{i}\left\{\nu_{i j}\right\} \leq \nu_{\alpha} \leq \max _{j}$ $\max _{i}\left\{\nu_{i j}\right\}$. Thus, by definition of score function, we get:

$$
\begin{aligned}
S(\alpha)= & \mu_{\alpha}-\nu_{\alpha} \leq \max _{j} \max _{i}\left\{\mu_{i j}\right\} \\
& -\min _{j} \min _{i}\left\{\nu_{i j}\right\}=S\left(F_{e_{i j}}^{+}\right), \\
S(\alpha)= & \mu_{\alpha}-\nu_{\alpha} \geq \min _{j} \min _{i}\left\{\mu_{i j}\right\} \\
& -\max _{j} \max _{i}\left\{\nu_{i j}\right\}=S\left(F_{e_{i j}}^{-}\right) .
\end{aligned}
$$

In that direction, the following cases are considered:

- Case 1: If $S\left(F_{e_{i j}}\right)<S\left(F_{e_{i j}}^{+}\right)$and $S\left(F_{e_{i j}}\right)>S\left(F_{e_{i j}}^{-}\right)$, then, by comparison laws between two IFSNs, we have:

$$
F_{e_{i j}}^{-}<\operatorname{IFSWA} A\left(F_{e_{11}}, F_{e_{12}}, \ldots, F_{e_{n m}}\right)<F_{e_{i j}}^{+} .
$$

- Case 2: If $S\left(F_{e_{i j}}\right)=S\left(F_{e_{i j}}^{+}\right)$, i.e. $\mu_{\alpha}-\nu_{\alpha}=$ $\max _{j} \max _{i}\left\{\mu_{i j}\right\}-\min _{j} \min _{i}\left\{\nu_{i j}\right\}$, then by the above inequalities, we have $\mu_{\alpha}=\max _{j} \max _{i}\left\{\mu_{i j}\right\}$ and $\nu_{\alpha}=$ $\min _{j} \min _{i}\left\{\nu_{i j}\right\}$. Thus:

$$
\begin{aligned}
H(\alpha) & =\mu_{\alpha}+\nu_{\alpha}=\max _{j} \max _{i}\left\{\mu_{i j}\right\}+\min _{j} \min _{i}\left\{\nu_{i j}\right\} \\
& =H\left(F_{e_{i j}}^{+}\right),
\end{aligned}
$$

then, by comparison laws between two IFSNs, we have:

$$
\operatorname{IFSWA} A\left(F_{e_{11}}, F_{e_{12}}, \ldots, F_{e_{n m}}\right)=F_{e_{i j}}^{+} .
$$

- Case 3: If $S\left(F_{e_{i j}}\right)=S\left(F_{e_{i j}}^{-}\right)$i.e. $\mu_{\alpha}-\nu_{\alpha}=$ $\min _{j} \min _{i}\left\{\mu_{i j}\right\}-\max _{j} \max _{i}\left\{\nu_{i j}\right\}$, then by the above $\stackrel{j}{i} \stackrel{i}{i n e q u a l i t i e s,}$ we have $\mu_{\alpha}=\min _{j} \min _{i}\left\{\mu_{i j}\right\}$ and $\nu_{\alpha}=$ $\max _{j} \max _{i}\left\{\nu_{i j}\right\}$. Thus:

$$
\begin{aligned}
H(\alpha) & =\mu_{\alpha}+\nu_{\alpha}=\min _{j} \min _{i}\left\{\mu_{i j}\right\}+\max _{j} \max _{i}\left\{\nu_{i j}\right\} \\
& =H\left(F_{e_{i j}}^{-}\right),
\end{aligned}
$$

then, it follows that:

$$
\operatorname{IFSWA}\left(F_{e_{11}}, F_{e_{12}}, \ldots, F_{e_{n m}}\right)=F_{e_{i j}}^{-} .
$$

Hence, property holds. 
Property 3.3 (Shift-invariance). If $F_{e}=\langle\mu, \nu\rangle$ is another IFSN, then:

$$
\begin{array}{r}
\operatorname{IFSWA}\left(F_{e_{11}} \oplus F_{e}, F_{e_{12}} \oplus F_{e}, \ldots, F_{e_{n m}} \oplus F_{e}\right) \\
=I F S W A\left(F_{e_{11}}, F_{e_{12}}, \ldots, F_{e_{n m}}\right) \oplus F_{e} .
\end{array}
$$

Proof. As $F_{e}$ and $F_{e_{i j}}$ are IFSNs, so $F_{e} \oplus F_{e_{i j}}=\langle 1$ $\left.-(1-\mu)\left(1-\mu_{i j}\right), \nu \nu_{i j}\right\rangle$. Therefore:

$$
\begin{aligned}
I F S W A & \left(F_{e_{11}} \oplus F_{e}, F_{e_{12}} \oplus F_{e}, \ldots, F_{e_{n m}} \oplus F_{e}\right) \\
= & \bigoplus_{j=1}^{m} \eta_{j}\left(\bigoplus_{i=1}^{n} \xi_{i}\left(F_{e_{i j}} \oplus F_{e}\right)\right) \\
= & \left\langle 1-\prod_{j=1}^{m}\left(\prod_{i=1}^{n}\left(1-\mu_{i j}\right)^{\xi_{i}}(1-\mu)^{\xi_{i}}\right)^{\eta_{j}},\right. \\
& \left.\prod_{j=1}^{m}\left(\prod_{i=1}^{n} \nu_{i j}^{\xi_{i}} \nu^{\xi_{i}}\right)^{\eta_{j}}\right\rangle \\
= & \left\langle 1-(1-\mu) \prod_{j=1}^{m}\left(\prod_{i=1}^{n}\left(1-\mu_{i j}\right)^{\xi_{i}}\right)^{\eta_{j}},\right. \\
& \left.\nu \prod_{j=1}^{m}\left(\prod_{i=1}^{n} \nu_{i j}^{\xi_{i}}\right)^{\eta_{j}}\right\rangle \\
= & \left\langle 1-\prod_{j=1}^{m}\left(\prod_{i=1}^{n}\left(1-\mu_{i j}\right)^{\xi_{i}}\right)^{\eta_{j}},\right. \\
& \left.\prod_{j=1}^{m}\left(\prod_{i=1}^{n}\left(\nu_{i j}\right)^{\xi_{i}}\right)^{\eta_{j}}\right\rangle \oplus\langle\mu, \nu\rangle \\
= & I F S W A\left(F_{e_{11}}, F_{e_{12}}, \ldots, F_{e_{n m}}\right) \oplus F_{e} .
\end{aligned}
$$

Hence, the property holds.

Property 3.4 (Homogeneity). For any real number $\lambda>0$, we have:

$$
\begin{aligned}
\operatorname{IFSWA} & \left(\lambda F_{e_{11}}, \lambda F_{e_{12}}, \ldots, \lambda F_{e_{n m}}\right) \\
& =\lambda \operatorname{IFSWA}\left(F_{e_{11}}, F_{e_{12}}, \ldots, F_{e_{n m}}\right) .
\end{aligned}
$$

Proof. For IFSN $F_{e_{i j}}$ and real number $\lambda>0$, we have $\lambda F_{e_{i j}}=\left\langle 1-\left(1-\mu_{i j}\right)^{\lambda}, \nu_{i j}^{\lambda}\right\rangle$. Therefore:

$$
\begin{aligned}
& \operatorname{IFSWA} A\left.\lambda F_{e_{11}}, \lambda F_{e_{12}}, \ldots, \lambda F_{e_{n m}}\right) \\
&=\left\langle 1-\prod_{j=1}^{m}\left(\prod_{i=1}^{n}\left(1-\mu_{i j}\right)^{\lambda \xi_{i}}\right)^{\eta_{j}},\right.
\end{aligned}
$$

$$
\begin{aligned}
& \left.\prod_{j=1}^{m}\left(\prod_{i=1}^{n} \nu_{i j}^{\lambda \xi_{i}}\right)^{\eta_{j}}\right\rangle \\
= & \left\langle 1-\left(\prod_{j=1}^{m}\left(\prod_{i=1}^{n}\left(1-\mu_{i j}\right)^{\xi_{i}}\right)^{\eta_{j}}\right)^{\lambda},\right. \\
& \left.\left(\prod_{j=1}^{m}\left(\prod_{i=1}^{n} \nu_{i j}^{\xi_{i}}\right)^{\eta_{j}}\right)^{\lambda}\right\rangle \\
= & \lambda \operatorname{IFSWA} A\left(F_{e_{11}}, F_{e_{12}}, \ldots, F_{e_{n m}}\right) .
\end{aligned}
$$

Hence, the property holds.

\subsection{Intuitionistic Fuzzy Soft Weighted Geometric (IFSWG) operator}

Definition 3.3. Let $F_{e_{i j}}=\left\langle\mu_{i j}, \nu_{i j}\right\rangle$, be an IFSN and $\eta_{j}, \xi_{i}$ be the standardized weight vectors for the parameters $e_{j}$ 's and experts $x_{i}$ 's respectively; IFSWG operator is defined as:

$$
\operatorname{IFSWG}\left(F_{e_{11}}, F_{e_{12}}, \ldots, F_{e_{n m}}\right)=\bigotimes_{j=1}^{m}\left(\bigotimes_{i=1}^{n} F_{e_{i j}}^{\xi_{i}}\right)^{\eta_{j}} .
$$

Theorem 3.2. The value obtained by using IFSWG operator is still an IFSN and given by:

$$
\begin{gathered}
\operatorname{IFSWG}\left(F_{e_{11}}, F_{e_{12}}, \ldots, F_{e_{n m}}\right)=\left\langle\prod_{j=1}^{m}\left(\prod_{i=1}^{n} \mu_{i j}^{\xi_{i}}\right)^{\eta_{j}},\right. \\
\left.1-\prod_{j=1}^{m}\left(\prod_{i=1}^{n}\left(1-\nu_{i j}\right)^{\xi_{i}}\right)^{\eta_{j}}\right\rangle .
\end{gathered}
$$

Proof. For $n=1$, we have $\xi_{1}=1$; by operations law given in Definition 3.1, we have:

$$
\begin{aligned}
\operatorname{IF} & S W G\left(F_{e_{11}}, F_{e_{12}}, \ldots, F_{e_{1 m}}\right)=\bigotimes_{j=1}^{m} F_{e_{1 j}}^{\eta_{j}} \\
& =\left\langle\prod_{j=1}^{m} \mu_{1 j}^{\eta_{j}}, 1-\prod_{j=1}^{m}\left(1-\nu_{1 j}\right)^{\eta_{j}}\right\rangle \\
& =\left\langle\prod_{j=1}^{m}\left(\prod_{i=1}^{1} \mu_{i j}^{\xi_{i}}\right)^{\eta_{j}}, 1-\prod_{j=1}^{m}\left(\prod_{i=1}^{1}\left(1-\nu_{i j}\right)^{\xi_{i}}\right)^{\eta_{j}}\right\rangle .
\end{aligned}
$$

For $m=1$, we have $\eta_{1}=1$; and then from Definition 3.3 , we get:

$$
\begin{gathered}
\operatorname{IFSWG}\left(F_{e_{11}}, F_{e_{21}}, \ldots, F_{e_{n 1}}\right)=\bigotimes_{i=1}^{n} F_{e_{i 1}}^{\xi_{i}} \\
=\left\langle\prod_{i=1}^{n} \mu_{i 1}^{\xi_{i}}, 1-\prod_{i=1}^{n}\left(1-\nu_{i 1}\right)^{\xi_{i}}\right\rangle
\end{gathered}
$$




$$
=\left\langle\prod_{j=1}^{1}\left(\prod_{i=1}^{n} \mu_{i j}^{\xi_{i}}\right)^{\eta_{j}}, 1-\prod_{j=1}^{1}\left(\prod_{i=1}^{n}\left(1-\nu_{i j}\right)^{\xi_{i}}\right)^{\eta_{j}}\right\rangle .
$$

Assume the result holds for $m=k_{1}+1, n=k_{2}$ and $m=k_{1}, n=k_{2}+1$, i.e.:

$$
\begin{aligned}
\bigotimes_{j=1}^{k_{1}+1}\left(\bigotimes_{i=1}^{k_{2}} F_{e_{i j}}^{\xi_{i}}\right)^{\eta_{j}}= & \left\langle\prod_{j=1}^{k_{1}+1}\left(\prod_{i=1}^{k_{2}} \mu_{i j}^{\xi_{i}}\right)^{\eta_{j}}, 1\right. \\
& \left.-\prod_{j=1}^{k_{1}+1}\left(\prod_{i=1}^{k_{2}}\left(1-\nu_{i j}\right)^{\xi_{i}}\right)^{\eta_{j}}\right\rangle
\end{aligned}
$$

and:

$$
\begin{aligned}
\bigotimes_{j=1}^{k_{1}}\left(\bigotimes_{i=1}^{k_{2}+1} F_{e_{i j}}^{\xi_{i}}\right)^{\eta_{j}}= & \left\langle\prod_{j=1}^{k_{1}}\left(\prod_{i=1}^{k_{2}+1} \mu_{i j}^{\xi_{i}}\right)^{\eta_{j}}, 1\right. \\
& \left.-\prod_{j=1}^{k_{1}}\left(\prod_{i=1}^{k_{2}+1}\left(1-\nu_{i j}\right)^{\xi_{i}}\right)^{\eta_{j}}\right\rangle
\end{aligned}
$$

Now, for $m=k_{1}+1, n=k_{2}+1$ :

$$
\begin{aligned}
\bigotimes_{j=1}^{k_{1}+1} & \left(\bigotimes_{i=1}^{k_{2}+1} F_{e_{i j}}^{\xi_{i}}\right)^{\eta_{j}}=\bigotimes_{j=1}^{k_{1}+1}\left(\bigotimes_{i=1}^{k_{2}} F_{e_{i j}}^{\xi_{i}} \otimes F_{e_{\left(k_{2}+1\right) j}}^{\xi_{k_{2}+1}}\right)^{\eta_{j}} \\
= & \bigotimes_{j=1}^{k_{1}+1}\left(\bigotimes_{i=1}^{k_{2}} F_{e_{i j}}^{\xi_{i}}\right)^{\eta_{j}} \bigotimes_{j=1}^{k_{1}+1}\left(F_{e_{\left(k_{2}+1\right) j}}^{\xi_{k_{2}+1}}\right)^{\eta_{j}} \\
= & \left\langle\prod_{j=1}^{k_{1}+1}\left(\prod_{i=1}^{k_{2}} \mu_{i j}^{\xi_{i}}\right)^{\eta_{j}} \otimes \prod_{j=1}^{k_{1}+1}\left(\mu_{\left(k_{2}+1\right) j}^{\xi_{k_{2}+1}}\right)^{\eta_{j}}, 1\right. \\
& -\prod_{j=1}^{k_{1}+1}\left(\prod_{i=1}^{k_{2}}\left(1-\nu_{i j}\right)^{\xi_{i}}\right)^{\eta_{j}} \otimes 1 \\
& \left.-\prod_{j=1}^{k_{1}+1}\left(\left(1-\nu_{\left(k_{2}+1\right) j}\right)^{\xi_{k_{2}+1}}\right)^{\eta_{j}}\right\rangle \\
= & \left\langle\prod_{j=1}^{k_{1}+1}\left(\prod_{i=1}^{k_{2}+1} \mu_{i j}^{\xi_{i}}\right)^{\eta_{j}}, 1-\prod_{j=1}^{k_{1}+1}\left(\prod_{i=1}^{k_{2}+1}\left(1-\nu_{i j}\right)^{\xi_{i}}\right)^{\eta_{j}}\right\rangle
\end{aligned}
$$

Therefore, Eq. (6) is true for $m=k_{1}+1$ and $n=$ $k_{2}+1$ and hence, by induction, it holds for all positive integers $m, n \geq 1$.

Since $0 \leq \nu_{i j} \leq 1 \Leftrightarrow 0 \leq \prod_{i=1}^{n}\left(1-\nu_{i j}\right)^{\xi_{i}} \leq 1$, $\Leftrightarrow 0 \leq \prod_{j=1}^{m}\left(\prod_{i=1}^{n}\left(1-\nu_{i j}\right)^{\xi_{i}}\right)^{\eta_{j}} \leq 1,0 \leq 1-\prod_{j=1}^{m}\left(\prod_{i=1}^{n}(1-\right.$ $\left.\left.\nu_{i j}\right)^{\xi_{i}}\right)^{\eta_{j}} \leq 1$. On the other hand, $0 \leq \mu_{i j} \leq 1 \Leftrightarrow 0 \leq$

$$
\begin{aligned}
\prod_{i=1}^{n} \mu_{i j}^{\xi_{i}} \leq 1 \Leftrightarrow 0 \leq & \prod_{j=1}^{m}\left(\prod_{i=1}^{n} \mu_{i j}^{\xi_{i}}\right)^{\eta_{j}} \leq 1 . \text { Finally: } \\
\prod_{j=1}^{m}\left(\prod_{i=1}^{n} \mu_{i j}^{\xi_{i}}\right)^{\eta_{j}} & +1-\prod_{j=1}^{m}\left(\prod_{i=1}^{n}\left(1-\nu_{i j}\right)^{\xi_{i}}\right)^{\eta_{j}} \\
& \leq 1-\prod_{j=1}^{m}\left(\prod_{i=1}^{n}\left(1-\nu_{i j}\right)^{\xi_{i}}\right)^{\eta_{j}} \\
& +\prod_{j=1}^{m}\left(\prod_{i=1}^{n}\left(1-\nu_{i j}\right)^{\xi_{i}}\right)^{\eta_{j}} \leq 1 .
\end{aligned}
$$

Thus, the aggregated value obtained by IFSWG operator is again an IFSN.

Example 3.2. Let $X=\left\{x_{1}, x_{2}, x_{3}, x_{4}\right\}$, the set of experts whose weight vector is $\xi=(0.1,0.2,0.4,0.3)^{T}$, give their preferences for describing the "attractiveness of a house" on certain parameters $E=\left\{e_{1}, e_{2}, e_{3}\right\}$ with weight $\eta=(0.3,0.5,0.2)^{T}$. The experts give their preference values in terms of IFSNs, which are represented in terms of decision matrix $(F, E)$ as:

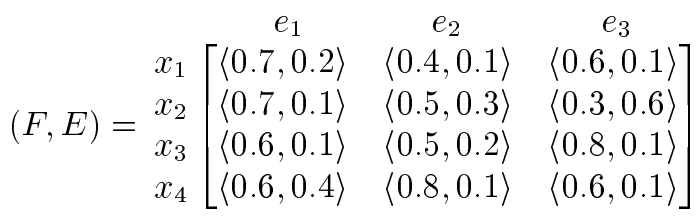

Thus, by using IFSWG operator as defined in Eq. (6), we get:

$$
I F S W G\left(F_{e_{11}}, F_{e_{12}}, \ldots, F_{e_{43}}\right)=\langle 0.5866,0.2027\rangle .
$$

Property 3.5. The IFSWG operator also satisfies the following properties, as similar to IFSWA operator, for IFSNs $F_{e_{i j}}=\left\langle\mu_{i j}, \nu_{i j}\right\rangle$ :

(P1) (Idempotency) If all $F_{e_{i j}}=F_{e}=\langle\mu, \nu\rangle$ (say), then $\operatorname{IFSWG}\left(F_{e_{11}}, F_{e_{12}}, \ldots, F_{e_{n m}}\right)=F_{e}$.

(P2) (Boundedness) If $F_{e_{i j}}^{-}=\left\langle\min _{j} \min _{i}\left\{\mu_{i j}\right\}, \max _{j}\right.$ $\left.\max _{i}\left\{\nu_{i j}\right\}\right\rangle$ and $F_{e_{i j}}^{+}=\left\langle\max _{j} \max _{i}\left\{\mu_{i j}\right\}, \min _{j}\right.$ $\left.\min _{i}\left\{\nu_{i j}\right\}\right\rangle$ then $F_{e_{i j}}^{-} \leq \operatorname{IFSWG}\left(F_{e_{11}}, F_{e_{12}}, \ldots\right.$, $\left.F_{e_{n m}}\right) \leq F_{e_{i j}}^{+}$.

(P3) (Shift-invariance) If $F_{e}=\langle\mu, \nu\rangle$ is another IFSN, then:

$$
\begin{array}{r}
I F S W G\left(F_{e_{11}} \otimes F_{e}, F_{e_{12}} \otimes F_{e}, \ldots, F_{e_{n m}} \otimes F_{e}\right) \\
=I F S W G\left(F_{e_{11}}, F_{e_{12}}, \ldots, F_{e_{n m}}\right) \otimes F_{e} .
\end{array}
$$

(P4) (Homogeneity) For a real number $\lambda>0$, we have:

$$
\begin{aligned}
\operatorname{IFSWG}\left(F_{e_{11}}^{\lambda}, F_{e_{12}}^{\lambda}, \ldots, F_{e_{n m}}^{\lambda}\right) \\
=\left(I F S W G\left(F_{e_{11}}, F_{e_{12}}, \ldots, F_{e_{n m}}\right)\right)^{\lambda} .
\end{aligned}
$$




\section{Decision-making approach based on proposed operators}

In this section, an approach for MCDM method has been presented based on the proposed operators followed by the practical example for showing their effectiveness.

\subsection{An approach based on proposed operators}

Let $p=\left\{p_{1}, p_{2}, \ldots, p_{t}\right\}$ be the set of $t$ different alternatives, which are going to be evaluated by the set of $n$ experts, $x_{1}, x_{2}, \ldots, x_{n}$, under the constraints of $m$ parameters, $E=\left\{e_{1}, e_{2}, \ldots, e_{m}\right\}$. Assume that weight vectors of the experts and parameters are $x_{i}$ and $e_{j}$, respectively, where $\xi=\left(\xi_{1}, \xi_{2}, \ldots, \xi_{n}\right)^{T}$ such that $\xi_{i} \in(0,1], \sum_{i=1}^{n} \xi_{i}=1$, and $\eta=\left(\eta_{1}, \eta_{2}, \ldots, \eta_{m}\right)^{T}$ such that $\eta_{j} \in(0,1]$ and $\sum_{j=1}^{m} \eta_{j}=1$. These $n$ experts give their preferences for the $t$ alternatives in terms of IFSNs $F_{e_{i j}}=\left\langle\mu_{i j}, \nu_{i j}\right\rangle$ such that $\mu_{i j}+\nu_{i j} \leq 1$; hence, the overall collective decision matrix is expressed as $D=\left(F_{e_{i j}}\right)_{n \times m}$. Based on these preferences values, the aggregated IFSN $\chi_{k}$ for alternative $p_{k}(k=1,2, \ldots, t)$ is $\chi_{k}=\left\langle\mu_{k}, \nu_{k}\right\rangle$ by using weighted averaging or geometric operators as given in Eq. (3) or Eq. (6). Finally, the score function of the aggregated IFSN $F_{e_{k}}(k=$ $1,2, \ldots, t)$ is utilized to rank the alternatives.

The above-mentioned technique has been summarized in the following steps:

- Step 1. Collect the information related to each alternative under the different parameters and arranged them in the form of intuitionistic fuzzy soft matrix, $D=\left\langle\mu_{i j}, \nu_{i j}\right\rangle_{n \times m}$, as:

$$
D_{n \times m}=\left[\begin{array}{cccc}
\left\langle\mu_{11}, \nu_{11}\right\rangle & \left\langle\mu_{12}, \nu_{12}\right\rangle & \ldots & \left\langle\mu_{1 m}, \nu_{1 m}\right\rangle \\
\left\langle\mu_{21}, \nu_{21}\right\rangle & \left\langle\mu_{22}, \nu_{22}\right\rangle & \ldots & \left\langle\mu_{2 m}, \nu_{2 m}\right\rangle \\
\vdots & \vdots & \ddots & \vdots \\
\left\langle\mu_{n 1}, \nu_{n 1}\right\rangle & \left\langle\mu_{n 2}, \nu_{n 2}\right\rangle & \ldots & \left\langle\mu_{n m}, \nu_{n m}\right\rangle
\end{array}\right]
$$

- Step 2. Normalize this collective information decision matrix by transforming the rating values of cost type parameters into the benefit type parameters, if any, by using the normalization formula [5]:

$$
r_{i j}= \begin{cases}F_{e_{i j}}^{c} & ; \quad \text { for cost type parameters } \\ F_{e_{i j}} & ; \quad \text { for benefit type parameters }\end{cases}
$$

where $F_{e_{i j}}^{c}=\left\langle\nu_{i j}, \mu_{i j}\right\rangle$ is the complement to $F_{e_{i j}}=$ $\left\langle\mu_{i j}, \nu_{i j}\right\rangle$.

- Step 3. Aggregate the IFSNs $F_{e_{i j}}(i=1,2, \ldots$, $n ; j=1,2, \ldots, m)$ for each alternative $p_{k}(k=$ $1,2, \ldots, t)$ into collective decision matrix $\chi_{k}$ by the proposed IFSWA (or IFSWG) operator.

- Step 4. By using Eq. (1), get the score value of $\chi_{k}$ for each alternative $p_{k}(k=1,2, \ldots, t)$.

- Step 5. Rank the alternatives $p_{k}(k=1,2, \ldots, t)$ and select the best one(s).

- Step 6. End.

\subsection{Practical example}

The above decision-making procedure has been illustrated with a practical example of medical diagnosis. The panel of five doctors, $d_{1}, d_{2}, d_{3}, d_{4}$, and $d_{5}$ whose weight vector is $\xi=(0.2,0.15,0.2,0.3,0.15)^{T}$, will give their opinion for four patients $p_{1}, p_{2}, p_{3}$, and $p_{4}$ under some parameters $E=\left\{\right.$ "Temperature $\left(e_{1}\right)$ ", "Headache $\left(e_{2}\right)$ ", "Stomach pain $\left(e_{3}\right)$ ", "Cough $\left(e_{4}\right)$ ", "Chest pain $\left(e_{5}\right)$ " $\}$ with weight vector $\eta=(0.2,0.1$, $0.3,0.15,0.25)^{T}$. The following are the steps followed for finding the illness of the patients by using the proposed approach.

\subsubsection{By IFSWA operator}

- Step 1. These five doctors $d_{i}$ will evaluate the illness of four patients in terms of IFSNs; parameters and their rating values are summarized in Tables 1-4, respectively.

- Step 2. Since all the parameters are of the same type, so there is no need for normalization.

- Step 3. The different opinions of the doctors for each patient $p_{k}(k=1,2,3,4)$ are aggregated by using Eq. (3); hence, we get:

$$
\begin{array}{ll}
\chi_{1}=\langle 0.5101,0.1877\rangle, & \chi_{2}=\langle 0.5060,0.2006\rangle, \\
\chi_{3}=\langle 0.4449,0.1932\rangle, & \chi_{4}=\langle 0.5193,0.1707\rangle .
\end{array}
$$

- Step 4. The score values are:

$$
\begin{aligned}
& S\left(\chi_{1}\right)=0.3224, \quad S\left(\chi_{2}\right)=0.3054, \\
& S\left(\chi_{3}\right)=0.2517, \quad S\left(\chi_{4}\right)=0.3486,
\end{aligned}
$$

thus, their ranking is $S\left(\chi_{4}\right) \succ S\left(\chi_{1}\right) \succ S\left(\chi_{2}\right) \succ$ $S\left(\chi_{3}\right)$.

Table 1. Intuitionistic fuzzy soft matrix for the patient $p_{1}$.

\begin{tabular}{cccccc}
\hline & Temperature & Headache & Stomach pain & Cough & Chest pain \\
\hline$d_{1}$ & $\langle 0.3,0.4\rangle$ & $\langle 0.5,0.1\rangle$ & $\langle 0.6,0.2\rangle$ & $\langle 0.7,0.1\rangle$ & $\langle 0.6,0.2\rangle$ \\
$d_{2}$ & $\langle 0.6,0.1\rangle$ & $\langle 0.6,0.2\rangle$ & $\langle 0.2,0.4\rangle$ & $\langle 0.5,0.1\rangle$ & $\langle 0.7,0.3\rangle$ \\
$d_{3}$ & $\langle 0.5,0.1\rangle$ & $\langle 0.7,0.2\rangle$ & $\langle 0.5,0.4\rangle$ & $\langle 0.2,0.2\rangle$ & $\langle 0.4,0.2\rangle$ \\
$d_{4}$ & $\langle 0.2,0.4\rangle$ & $\langle 0.5,0.1\rangle$ & $\langle 0.6,0.1\rangle$ & $\langle 0.4,0.1\rangle$ & $\langle 0.6,0.2\rangle$ \\
$d_{5}$ & $\langle 0.6,0.1\rangle$ & $\langle 0.3,0.4\rangle$ & $\langle 0.4,0.3\rangle$ & $\langle 0.6,0.1\rangle$ & $\langle 0.5,0.2\rangle$ \\
\hline
\end{tabular}


Table 2. Intuitionistic fuzzy soft matrix for the patient $p_{2}$.

\begin{tabular}{cccccc}
\hline & Temperature & Headache & Stomach pain & Cough & Chest pain \\
\hline$d_{1}$ & $\langle 0.4,0.3\rangle$ & $\langle 0.5,0.1\rangle$ & $\langle 0.6,0.2\rangle$ & $\langle 0.7,0.1\rangle$ & $\langle 0.7,0.2\rangle$ \\
$d_{2}$ & $\langle 0.6,0.1\rangle$ & $\langle 0.5,0.3\rangle$ & $\langle 0.4,0.3\rangle$ & $\langle 0.4,0.3\rangle$ & $\langle 0.4,0.1\rangle$ \\
$d_{3}$ & $\langle 0.5,0.3\rangle$ & $\langle 0.5,0.1\rangle$ & $\langle 0.5,0.3\rangle$ & $\langle 0.3,0.2\rangle$ & $\langle 0.6,0.2\rangle$ \\
$d_{4}$ & $\langle 0.5,0.3\rangle$ & $\langle 0.7,0.3\rangle$ & $\langle 0.4,0.2\rangle$ & $\langle 0.5,0.1\rangle$ & $\langle 0.5,0.2\rangle$ \\
$d_{5}$ & $\langle 0.4,0.2\rangle$ & $\langle 0.5,0.2\rangle$ & $\langle 0.3,0.3\rangle$ & $\langle 0.6,0.1\rangle$ & $\langle 0.4,0.2\rangle$ \\
\hline
\end{tabular}

Table 3. Intuitionistic fuzzy soft matrix for the patient $p_{3}$.

\begin{tabular}{cccccc}
\hline & Temperature & Headache & Stomach pain & Cough & Chest pain \\
\hline$d_{1}$ & $\langle 0.4,0.3\rangle$ & $\langle 0.5,0.4\rangle$ & $\langle 0.5,0.2\rangle$ & $\langle 0.6,0.1\rangle$ & $\langle 0.4,0.2\rangle$ \\
$d_{2}$ & $\langle 0.5,0.1\rangle$ & $\langle 0.3,0.2\rangle$ & $\langle 0.3,0.2\rangle$ & $\langle 0.4,0.2\rangle$ & $\langle 0.3,0.2\rangle$ \\
$d_{3}$ & $\langle 0.5,0.3\rangle$ & $\langle 0.5,0.1\rangle$ & $\langle 0.4,0.2\rangle$ & $\langle 0.2,0.2\rangle$ & $\langle 0.5,0.4\rangle$ \\
$d_{4}$ & $\langle 0.5,0.1\rangle$ & $\langle 0.4,0.5\rangle$ & $\langle 0.3,0.2\rangle$ & $\langle 0.7,0.2\rangle$ & $\langle 0.3,0.2\rangle$ \\
$d_{5}$ & $\langle 0.7,0.1\rangle$ & $\langle 0.4,0.6\rangle$ & $\langle 0.4,0.2\rangle$ & $\langle 0.3,0.1\rangle$ & $\langle 0.6,0.1\rangle$ \\
\hline
\end{tabular}

Table 4. Intuitionistic fuzzy soft matrix for the patient $p_{4}$.

\begin{tabular}{cccccc}
\hline & Temperature & Headache & Stomach pain & Cough & Chest pain \\
\hline$d_{1}$ & $\langle 0.3,0.4\rangle$ & $\langle 0.8,0.1\rangle$ & $\langle 0.7,0.1\rangle$ & $\langle 0.4,0.3\rangle$ & $\langle 0.2,0.3\rangle$ \\
$d_{2}$ & $\langle 0.5,0.1\rangle$ & $\langle 0.4,0.2\rangle$ & $\langle 0.4,0.2\rangle$ & $\langle 0.6,0.1\rangle$ & $\langle 0.2,0.6\rangle$ \\
$d_{3}$ & $\langle 0.2,0.1\rangle$ & $\langle 0.4,0.2\rangle$ & $\langle 0.5,0.4\rangle$ & $\langle 0.4,0.2\rangle$ & $\langle 0.5,0.2\rangle$ \\
$d_{4}$ & $\langle 0.7,0.2\rangle$ & $\langle 0.5,0.1\rangle$ & $\langle 0.6,0.1\rangle$ & $\langle 0.4,0.1\rangle$ & $\langle 0.7,0.1\rangle$ \\
$d_{5}$ & $\langle 0.5,0.2\rangle$ & $\langle 0.5,0.4\rangle$ & $\langle 0.4,0.2\rangle$ & $\langle 0.3,0.2\rangle$ & $\langle 0.7,0.1\rangle$ \\
\hline
\end{tabular}

- Step 5. Therefore, the patient $p_{4}$ has more illness than the others patients.

\subsubsection{IFSWG operator}

If we utilize IFSWG operator for the considered problem to find the illness of patients, the following steps should be taken:

- Step 1. Same as that of above.

- Step 2. All the parameters are of the same type, so there is no need of normalizing the data.

- Step 3. The aggregated rating values for each patient $p_{k}(k=1,2,3,4)$ by using Eq. (6) are:

$$
\begin{array}{ll}
\chi_{1}=\langle 0.4589,0.2251\rangle, & \chi_{2}=\langle 0.4808,0.2187\rangle, \\
\chi_{3}=\langle 0.4118,0.2223\rangle, \quad \chi_{4}=\langle 0.4561,0.2133\rangle .
\end{array}
$$

- Step 4. Their score values are:

$$
\begin{aligned}
& S\left(\chi_{1}\right)=0.2338, \quad S\left(\chi_{2}\right)=0.2621, \\
& S\left(\chi_{3}\right)=0.1895, \quad S\left(\chi_{4}\right)=0.2428 .
\end{aligned}
$$

- Step 5. Therefore, the patient $p_{2}$ has more illness than the others patients.

\subsection{Comparative studies}

In order to compare the proposed approach with some existing metrics, an analysis has been conducted based on different aggregation operators [39-42]. For this, first, the different parameters of the numbers are aggregated by using weighted averaging operator corresponding to weight vector $(0.2,0.1,0.3,0.15,0.25)^{T}$; the obtained aggregated decision matrix is summarized in Table 5. Now, based on this matrix, a comparative study is conducted based on different operators; their simultaneously results, for each patient, are shown in

Table 5. Aggregated intuitionistic fuzzy soft matrix for the patients.

\begin{tabular}{ccccc}
\hline & $\boldsymbol{p}_{\mathbf{1}}$ & $\boldsymbol{p}_{\mathbf{2}}$ & $\boldsymbol{p}_{\mathbf{3}}$ & $\boldsymbol{p}_{\mathbf{4}}$ \\
\hline$x_{1}$ & $\langle 0.5619,0.1932\rangle$ & $\langle 0.6046,0.1824\rangle$ & $\langle 0.4751,0.2095\rangle$ & $\langle 0.5161,0.2048\rangle$ \\
$x_{2}$ & $\langle 0.5261,0.2138\rangle$ & $\langle 0.4567,0.1830\rangle$ & $\langle 0.3605,0.1741\rangle$ & $\langle 0.4150,0.2065\rangle$ \\
$x_{3}$ & $\langle 0.4664,0.2144\rangle$ & $\langle 0.5027,0.2285\rangle$ & $\langle 0.4333,0.2407\rangle$ & $\langle 0.4251,0.2144\rangle$ \\
$x_{4}$ & $\langle 0.5007,0.1569\rangle$ & $\langle 0.4982,0.2036\rangle$ & $\langle 0.4325,0.1908\rangle$ & $\langle 0.6181,0.1149\rangle$ \\
$x_{5}$ & $\langle 0.4949,0.1889\rangle$ & $\langle 0.4194,0.2036\rangle$ & $\langle 0.5170,0.1473\rangle$ & $\langle 0.5111,0.1803\rangle$ \\
\hline
\end{tabular}


Table 6. Comparative studies.

\begin{tabular}{cccccc}
\hline \multirow{2}{*}{ Method } & \multicolumn{4}{c}{ Score values of the patients } & \multirow{2}{*}{ Ranking } \\
\cline { 2 - 5 } & $\boldsymbol{p}_{\mathbf{1}}$ & $\boldsymbol{p}_{\mathbf{2}}$ & $\boldsymbol{p}_{\mathbf{3}}$ & $\boldsymbol{p}_{\mathbf{4}}$ & \\
\hline Xu [39] & 0.3224 & 0.3054 & 0.2517 & 0.3486 & $p_{4} \succ p_{1} \succ p_{2} \succ p_{3}$ \\
Xu and Yager [40] & 0.3186 & 0.2976 & 0.2446 & 0.3292 & $p_{4} \succ p_{1} \succ p_{2} \succ p_{3}$ \\
Wang and Liu [41] & 0.3219 & 0.3042 & 0.2507 & 0.3460 & $p_{4} \succ p_{1} \succ p_{2} \succ p_{3}$ \\
Wang and Liu [42] & 0.3191 & 0.2988 & 0.2456 & 0.3320 & $p_{4} \succ p_{1} \succ p_{2} \succ p_{3}$ \\
\hline
\end{tabular}

Table 6. In this table, it is seen that the patient $p_{4}$ has more illness than others; these results overlap the proposed results. Thus, the proposed technique can be utilized by using the concepts of the soft set to solve the decision-making problem suitably in comparison with the other existing operators.

\section{Conclusion}

In this paper, we have established the two new aggregation operators, i.e. the IFSWA operator and the IFSWG operator, under the IFSS environment. For this, first, the algebraic structures of two IFSNs were presented and, corresponding to their operational laws, the aggregation operators were proposed. Moreover, some of their desirable properties were investigated in details. A decision-making method based on IFSWA and IFSWG operators was presented, in which preferences related to different alternatives were taken into account in terms of IFSNs. A comparative study was also performed for illustrating the approach. In the end, the proposed operators showed a more stable and practical nature for the decision makers during the aggregation process. From the obtained results, it was concluded that the proposed approach could be suitably utilized to solve the MCDM problem in today life situations. In the future work, we shall apply these operators to other fields such as mathematical programming, cluster analysis, big-data analysis, etc.

\section{Acknowledgments}

The first author (Rishu Arora) would like to thank the Department of Science \& Technology, New Delhi, India for providing financial support under WOS-A scheme wide file no. SR/WOS-A/PM-77/2016 for the preparation of this manuscript. The second author (Harish Garg) would like to thanks the Thapar Institute of Engineering \& Technology (Deemed University), Patiala for providing the financial support under SEED Money Grant wide letter no. TU/DORSP/57/1910.

\section{References}

1. Molodtsov, D. "Soft set theory - first results", Computer and Mathematics with Applications, 27(4-5), pp. 19-31 (1999).
2. Zadeh, L.A. "Fuzzy sets", Information and Control, 8, pp. 338-353 (1965).

3. Attanassov, K.T. "Intuitionistic fuzzy sets", Fuzzy Sets and Systems, 20, pp. 87-96 (1986).

4. Garg, H. "Generalized intuitionistic fuzzy interactive geometric interaction operators using Einstein t-norm and t-conorm and their application to decision making", Computer and Industrial Engineering, 101, pp. 53-69 (2016).

5. Xu, Z.S. and Hu, H. "Projection models for intuitionistic fuzzy multiple attribute decision making", International Journal of Information Technology and Decision Making, 9, pp. 267-280 (2010).

6. Wang, J.Q., Zhou, P., Li, K.J., Zhang, H.Y., and Chen, X.H. "Multi-criteria decision-making method based on normal intuitionistic fuzzy-induced generalized aggregation operator", TOP, 22(3), pp. 1103-1122 (2014).

7. Garg, H. "A new generalized pythagorean fuzzy information aggregation using Einstein operations and its application to decision making", International Journal of Intelligent Systems, 31(9), pp. 886-920 (2016).

8. Wang, J.Q., Han, Z.Q., and Zhang, H.Y. "Multi criteria group decision-making method based on intuitionistic interval fuzzy information", Group Decision and Negotiation, 23, pp. 715-733 (2014).

9. Garg, H. "A new generalized improved score function of interval-valued intuitionistic fuzzy sets and applications in expert systems", Applied Soft Computing, 38, pp. 988-999 (2016).

10. Garg, H. "A novel correlation coefficients between Pythagorean fuzzy sets and its applications to decisionmaking processes", International Journal of Intelligent Systems, 31(12), pp. 1234-1253 (2016).

11. Wang, P., Xu, X.-h., Wang, J.-q., and Cai, C.-G. "Some new operation rules and a new ranking method for interval-valued intuitionistic linguistic numbers", Journal of Intelligent and Fuzzy Systems, 32(1), pp. 1069-1078 (2017).

12. Wang, C. and Wang, J. "A multi-criteria decisionmaking method based on triangular intuitionistic fuzzy preference information", Intelligent Automation and Soft Computing, 22(3), pp. 473-482 (2016).

13. Garg, H. "Some series of intuitionistic fuzzy interactive averaging aggregation operators", SpringerPlus, 5(1), pp. 1-27 (2016). DOI: $10.1186 / \mathrm{s} 40064-016-2591-9$

14. Peng, J.J., Wang, J.Q., Wu, X.H., Zhang, H.Y., and Chen, X.H. "The fuzzy cross-entropy for intuitionistic 
hesitant fuzzy sets and its application in multi-criteria decision-making", International Journal of System Science, 46(13), pp. 2335-2350 (2015).

15. Garg, H., Agarwal, N., and Tripathi, A. "Some improved interactive aggregation operators under interval-valued intuitionistic fuzzy environment and its application to decision making process", Scientia Iranica, E, 24(5), pp. 2581-2604 (2017).

16. Maji, P.K., Biswas, R., and Roy, A.R. "Fuzzy soft sets", Journal of Fuzzy Mathematics, 9(3), pp. 589-602 (2001).

17. Maji, P.K., Biswas, R., and Roy, A. "Intuitionistic fuzzy soft sets", Journal of Fuzzy Mathematics, 9(3), pp. 677-692 (2001).

18. Cagman, N., Citak, F., and Enginoglu, S. "Fuzzy parameterized fuzzy soft set theory and its applications", Turkish Journal of Fuzzy System, 1(1), pp. 2135 (2010).

19. Cagman, N., Enginoglu, S., and Citak, F. "Fuzzy soft set theory and its applications", Iranian Journal of Fuzzy System, 8(3), pp. 137-147 (2011).

20. Cagman, N., and Deli, I. "Intuitionistic fuzzy parameterized soft set theory and its decision making", Applied Soft Computing, 28, pp. 109-113 (2015).

21. Alkhazaleh, S. and Salleh, A.R. "Fuzzy soft expert set and its application", Applied Mathematics, 5, pp. 13491368 (2014).

22. Yang, H.L. "Notes on generalized fuzzy soft sets", Journal of Mathematical Research and Exposition, 31(3), pp. 567-570 (2011).

23. Majumdar, P. and Samanta, S.K. "Generalized fuzzy soft sets", Computers and Mathematics with Applications, 59(4), pp. 1425-1432 (2010).

24. Agarwal, M., Biswas, K.K., and Hanmandlu, M. "Generalized intuitionistic fuzzy soft sets with applications in decision-making", Applied Soft Computing, 13, pp. 3552-3566 (2013).

25. Dinda, B., Bera, T., and Samanta, T.K. "Generalized intuitionistic fuzzy soft sets and its application in decision making" (2010).

http://arxiv.org/pdf/1010.2468.pdf

26. Agarwal, M., Hanmandlu, M., and Biswas, K.K. "Generalized intuitionistic fuzzy soft set and its application in practical medical diagnosis problem", in: IEEE International Conference on Fuzzy Systems (FUZZ), pp. 2972-2978 (2011).

27. Garg, H., Agarwal, N., and Tripathi, A. "Fuzzy number intuitionistic fuzzy soft sets and its properties", Journal of Fuzzy Set Valued Analysis, 2016(3), Article ID jfsva-00332, pp. 196-213 (2016).

28. Maji, P.K., Biswas, R., and Roy, A.K. "On intuitionistic fuzzy soft sets", Journal of Fuzzy Mathematics, 12(3), pp. 669-683 (2004).

29. Maji, P.K. "More on intuitionistic fuzzy soft sets", Lecture Notes in Computer Science, 5908, pp. 231-240 (2009).
30. Babitha, K.V. and John, S.J. "Hesistant fuzzy soft sets", Journal of New Results in Science, 3, pp. 98107 (2013).

31. Jiang, Y., Tang, Y., Chen, Q., Liu, H., and Tang, J. "Interval-valued intuitionistic fuzzy soft sets and their properties", Computers and Mathematics with Applications, 60(3), pp. 906-918 (2010).

32. Peng, X. and Yang, Y. "Algorithms for interval-valued fuzzy soft sets in stochastic multi-criteria decision making based on regret theory and prospect theory with combined weight", Applied Soft Computing, 54, pp. 415-430 (2017).

33. Yang, X., Lin, T.Y., Yang, J., Li, Y., and Yu, D. "Combination of interval-valued fuzzy set and soft set", Computer and Mathematics with Applications, 58(3), pp. 521-527 (2009).

34. Majumdar, P. and Samanta, S. "Similarity measure of soft sets", New Mathematics and Natural Computation, 4(1), pp. 1-12 (2008).

35. Rajarajeswari, P. and Dhanalakshmi, P. "Similarity measures of intuitionistic fuzzy soft sets and its application in medical diagnosis", International Journal of Mathematical Archive, 5(5), pp. 143-149 (2014).

36. Mukherjee, A. and Sarkar, S. "Similarity measures for interval-valued intuitionistic fuzzy soft sets and its application in medical diagnosis problem", New Trends in Mathematical Sciences, 2(3), pp. 159-165 (2014).

37. Khalid, A. and Abbas, M. "Distance measures and operations in intuitionistic and interval-valued intuitionistic fuzzy soft set theory", International Journal of Fuzzy Systems, 17(3), pp. 490-497 (2015).

38. Roy, A.R. and Maji, P.K. "A fuzzy soft set theoretic approach to decision making problems", Journal of Computational and Applied Mathematics, 203(2), pp. 412-418 (2007).

39. Xu, Z.S. "Intuitionistic fuzzy aggregation operators", IEEE Transaction of Fuzzy System, 15, pp. 1179-1187 (2007).

40. Xu, Z.S. and Yager, R.R. "Some geometric aggregation operators based on intuitionistic fuzzy sets", International Journal of General Systems, 35, pp. 417-433 (2006).

41. Wang, W. and Liu, X. "Intuitionistic fuzzy information aggregation using Einstein operations", IEEE Transactions on Fuzzy Systems, 20(5), pp. 923-938 (2012).

42. Wang, W.Z. and Liu, X.W. "Intuitionistic fuzzy geometric aggregation operators based on Einstein operations", International Journal of Intelligent Systems, 26, pp. 1049-1075 (2011).

\section{Biographies}

Rishu Arora received the Master degree in Mathematics from Department of Mathematics, Guru Nanak Dev University, Amritsar, India, in 2010. Presently, she is working towards her $\mathrm{PhD}$ degree in Thapar Institute 
of Engineering \& Technology (Deemed University), Patiala, Punjab, India. She has more than three years of teaching experience in different subjects of Engineering Mathematics. She has qualified GATE in 2012-2015. Her current research interests are in the areas of multi criteria decision-making, aggregation operator, and intuitionistic fuzzy soft set. She has published seven papers in the international reputed SCI journals.

Harish Garg is an Assistant Professor at Thapar Institute of Engineering \& Technology (Deemed University), Patiala, India. He received an MSc in Mathematics from Punjabi University, Patiala, India, in 2008 and a PhD in Applied Mathematics with speciality in reliability theory and soft computing techniques from Indian Institute of Technology, Roorkee, India, in 2013. His research interests are in the fields of computational intelligence, multicriteria decision making problems, reliability theory, optimization techniques, various nature-inspired algorithms, and fuzzy and intuitionistic fuzzy sets theories. Dr. Garg has authored/co-authored over 125 technical papers published in refereed International Journals. Also, he has published 6 book chapters. His biographical profile has been selected for inclusion in the International Who's Who of Professionals, Marquis Who's Who in the World, and Marquis Who's Who in Science and Engineering. He also severs as an editorial board member of various international journals. In the year 2016 and 2017, Dr. Garg was privileged an awarded outstanding reviewer for the journals Applied Soft Computing, Applied Mathematical Modeling and Engineering Applications of Artificial Intelligence, Elsevier. His Google citations are over 1750. For more details, visit http://sites.google.com/site/harishg58iitr/ 